\title{
Interventions to Improve Influenza and Pneumococcal Vaccination Rates Among Community-Dwelling Adults: A Systematic Review and Meta-Analysis
}

Darren Lau, BMSc ${ }^{1}$

Jia $H u, B A^{1}$

Sumit R. Majumdar, $M D, M P H^{2}$

Dale A. Storie, MLIS ${ }^{3}$

Sandra E. Rees, BScPharm ${ }^{4}$

Jeffrey A. Jobuson, $P b D^{1,4}$

'Department of Public Health Sciences, University of Alberta, Edmonton, Alberta, Canada

${ }^{2}$ Department of Medicine, Faculty of Medicine and Dentistry, University of Alberta Edmonton, Alberta, Canada

${ }^{3}$ John W. Scott Health Sciences Library, University of Alberta, Edmonton, Alberta, Canada

${ }^{4}$ Institute of Health Economics, Edmonton, Alberta, Canada

Conflicts of interest: authors report none.

\section{CORRESPONDING AUTHOR}

Jeffrey A. Johnson, PhD

2-040G Li Ka Shing Center for Health

Research Innovation

$8602112 \mathrm{St}$

University of Alberta

Edmonton, AB T6G 2E1

jeff.johnson@ualberta.ca

\begin{abstract}
PURPOSE Influenza and pneumococcal vaccination rates remain below national targets. We systematically reviewed the effectiveness of quality improvement interventions for increasing the rates of influenza and pneumococcal vaccinations among community-dwelling adults.

METHODS We included randomized and nonrandomized studies with a concurrent control group. We estimated pooled odds ratios using random effects models, and used the Downs and Black tool to assess the quality of included studies.

RESULTS Most studies involved elderly primary care patients. Interventions were associated with improvements in the rates of any vaccination (111 comparisons in 77 studies, pooled odds ratio $[\mathrm{OR}]=1.61,95 \% \mathrm{Cl}, 1.49-1.75$ ), and influenza (93 comparisons, 65 studies, $\mathrm{OR}=1.46,95 \% \mathrm{Cl}, 1.35-1.57)$ and pneumococcal (58 comparisons, 35 studies, $\mathrm{OR}=2.01,95 \% \mathrm{Cl}, 1.72-2.3$ ) vaccinations. Interventions that appeared effective were patient financial incentives (influenza only), audit and feedback (influenza only), clinician reminders, clinician financial incentives (influenza only), team change, patient outreach, delivery site changes (influenza only), clinician education (pneumococcus only), and case management (pneumococcus only). Patient outreach was more effective if personal contact was involved. Team changes were more effective where nurses administered influenza vaccinations independently. Heterogeneity in some pooled odds ratios was high, however, and funnel plots showed signs of potential publication bias. Study quality varied but was not associated with outcomes.
\end{abstract}

CONCLUSIONS Quality improvement interventions, especially those that assign vaccination responsibilities to nonphysician personnel or that activate patients through personal contact, can modestly improve vaccination rates in communitydwelling adults. To meet national policy targets, more-potent interventions should be developed and evaluated.

Ann Fam Med 2012;10:538-546. doi:10.1370/afm.1405.

\section{INTRODUCTION}

$\mathrm{I}$ nfluenza and pneumococcal disease are vaccine-preventable causes of morbidity and mortality. ${ }^{1-3}$ Clinical practice guidelines have recom-

mended routine influenza and pneumococcal vaccinations for elderly and nonelderly high-risk patients. ${ }^{4-6}$ More recently, influenza vaccinations have been recommended for all individuals older than 6 months. ${ }^{7}$ Even so, vaccination rates remain low. ${ }^{8,9}$

Studies of interventions for improving adult influenza and pneumococcal vaccination rates are numerous and have been synthesized in several systematic reviews. Jacobson and Szilagyi found that patient reminder and recall systems improved vaccination rates..$^{10}$ The US Preventive Services 
Task Force's (USPSTF) Community Guide to Preventive Services found supporting evidence for numerous interventions aimed at universally recommended vaccines ${ }^{11}$ and for combinations of multiple interventions for vaccines targeted to high-risk groups. ${ }^{12,13}$ Stone et al found that interventions involving organizational changes and teamwork were most effective for improving influenza or pneumococcal vaccination rates..$^{14}$ Most recently, Thomas et al found evidence of moderate quality that increasing community demand, vaccinating seniors during home visits, and deploying prevention facilitators working with health professionals improved influenza vaccination rates. ${ }^{15}$ Though important, these reviews have a variety of limitations. For example, Thomas et al included randomized controlled trials, most of which were graded low in quality. Consequently, the authors were able to recommend only that practitioners implement home visits (2 studies) and practice prevention facilitators (4 studies), to improve vaccination rates. ${ }^{15}$ The work of the USPSTF combined many vaccinations for different patient groups under targeted and universally recommended vaccinations. ${ }^{11,12}$ Stone et al, in their review of controlled clinical trials, examined the evidence more than a decade ago. We know of more than 50 additional studies that could be included, today. ${ }^{14}$

Because previous reviews may be of limited currency and breadth, we undertook a systematic review and meta-analysis of randomized and nonrandomized studies of the effectiveness of quality improvement interventions for improving adult influenza and pneumococcal vaccination rates in the community. Our review is intended to provide a comprehensive quantitative summary of the results achieved by previous quality improvement studies.

\section{METHODS}

\section{Study Selection and Data Extraction}

We searched medical literature databases, including MEDLINE, EMBASE, Cochrane Library, Web of Science, and 5 other databases, as well as the reference lists of previous reviews up to August 2010, for relevant studies (Supplemental Appendix A, Exhibit A.2, available at

English language studies published in peer-reviewed journals were included if they involved elderly adults or adults with chronic diseases, involved a quality improvement intervention (see below), featured a parallel control group, and reported influenza or pneumococcal vaccination rates. We focused exclusively on the community setting to maximize relevance to primary care. Studies reporting sufficient data to estimate log odds ratios (ORs) and standard errors were eligible for meta-analysis.

Two reviewers (D.L. and J.H.) selected studies and extracted data from each study in duplicate. Study quality was measured using the Downs and Black instrument, which assesses both randomized and nonrandomized studies on the same items. ${ }^{16}$ Disagreements were resolved by consensus; remaining disagreements were resolved by the senior authors (J.A.J. and S.R.M.).

\section{Data Synthesis}

We synthesized results by performing random-effects meta-analyses of log odds ratios. We stratified analyses by vaccination type and intervention category. To categorize the interventions, we modified the taxonomy developed by Shojania et al used to classify quality improvement strategies (http://dx.doi.org/10.1001/ jama.296.4.427) ${ }^{17,18}$ Comparisons were included in meta-analyses if the control group was usual care; a control intervention aimed at nonvaccination behaviors or a different intervention for improving vaccination rates if the intervention was provided to both study arms. When study arms contributed to more than 1 comparison in a meta-analysis, the vaccination rate numerator and denominator were divided among the comparisons to avoid counting patients more than once. We accounted for unit of analysis errors by adjusting standard errors for literature-based values of intracluster correlations. ${ }^{19,20}$ Although we reported all pooled odds ratios, we interpreted only those odds ratios comprised of 3 or more comparisons. Heterogeneity was characterized with $\mathrm{I}^{2}$ statistics. We explored heterogeneity by substratifying interventions with clear grounds for delineating strata and sufficient studies to divide into strata of 3 or more comparisons. Clinician reminders were stratified according to whether the reminder system was immunization specific or targeted a range of preventive care behaviors, and whether reminders were generated from patients' medical histories. Patient outreach interventions were stratified by communication medium. Finally, team change interventions were stratified by type of personnel involved and whether they administered vaccine independently.

The effects of Downs and Black scores and randomization on pooled odds ratios were examined by meta-regression. Finally, we tested for publication bias by visual inspection of funnel plots and by using Harbord's test. ${ }^{21}$ Harbord's test is an alternative to Egger's test that mitigates false positives in meta-analyses of odds ratios. Analyses were performed using Stata 11 (StataCorp LP). ${ }^{22}$

\section{RESULTS}

\section{Overview of Studies}

We included 106 and excluded 208 citations (Figure 1). Citations were most commonly excluded because 
Figure 1. Citation flow.

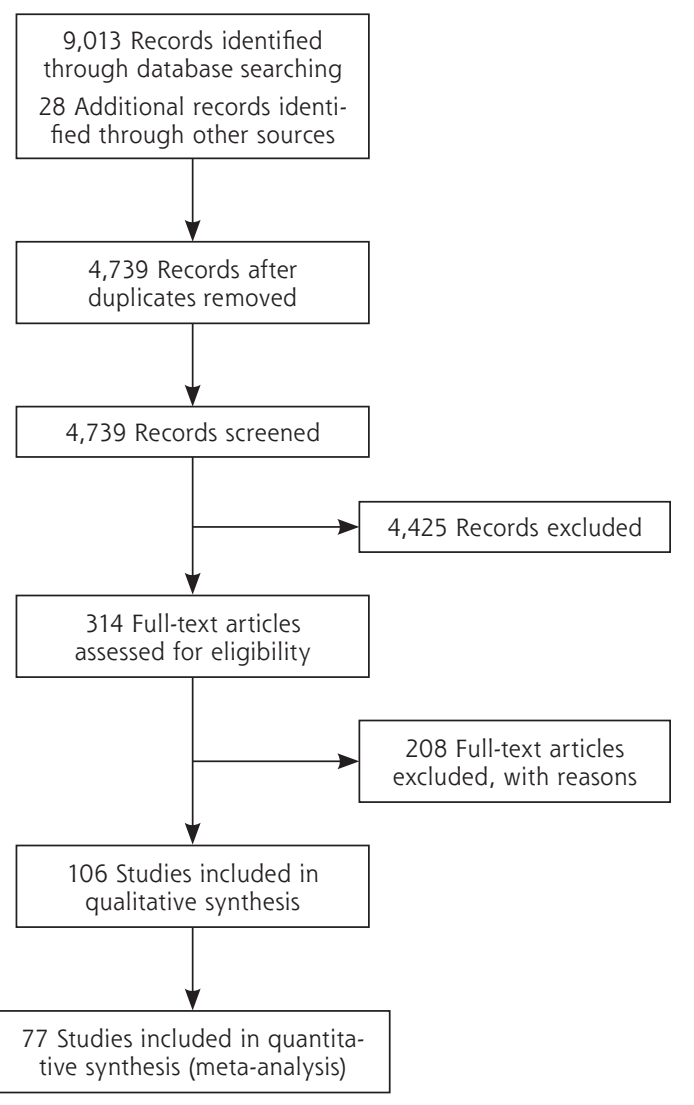

they lacked a concurrent control group $(\mathrm{n}=112,54 \%)$ or took place in hospital or in a nursing home $(\mathrm{n}=27$, 13\%) (Supplemental Appendix A, Exhibit A.3 and A.4). Interreviewer reliability for inclusion of electronic search citations was substantial ( $91 \%$ agreement, $\kappa=0.8$ ). The included studies featured 470,175 patients (Table 1 and Supplemental Appendix A, Exhibit A.5). Studies took place primarily in the United States (82 studies), Canada (9 studies), and the United Kingdom (6 studies). A range of settings was represented, including academic primary care practices (41 studies), community practices (21 studies), managed care organizations (13 studies), Medicare-affiliated organizations (11 studies), and Veterans Affairs medical centers ( 8 studies). A few studies intervened at nonclinical sites, such as senior centers or workplaces. Most stud- ies targeted the elderly for vaccination, either alone (54 studies), or in combination with high-risk nonelderly patients (27 studies).

\section{Quality of Included Studies}

Seventy-seven studies provided sufficient data for meta-analyses of odds ratios (Table 1 and Supplemental Appendix A, Exhibit A.6). Fifty-six studies (75\%) were randomized or quasi-randomized controlled trials. The remaining studies were controlled before-and-after ( 7 studies) and observational (12 studies) designs. The median Downs and Black scores ranged from 14 to 26, with a median score of 21 points (the median Downs and Black scores ranged from 14 to 26, with a median score of 21 points (total possible score $=32$, with greater scores indicating higher methodologic quality).

We examined individual items of the Downs and Black instrument (Supplemental Appendix B, http:// annfammed.org/content/10/6/538/suppl/DC1). The most important weaknesses were unit-ofanalyses errors and insufficient reporting and adjustment for potential confounders. We corrected for unit-of-analysis errors in 38 (51\%) of studies. Potential confounders included previous vaccination status, health status, and demographic characteristics. The proportion of studies reporting and accounting for these confounders, whether by showing that randomization achieved a balanced distribution of covariates or by statistical adjustment, was $60 \%$. Additional methodological weaknesses were lack of blinding of study subjects or assessors to intervention allocation and contamination, in which the intervention may have affected the treatment of nonintervention patients at
Table 1. Patients, Studies, and Comparisons by Quality Improvement Strategy

\begin{tabular}{lcccc}
\hline Quality Improvement & $\begin{array}{c}\text { No. of } \\
\text { Patients }\end{array}$ & $\begin{array}{c}\text { No. of } \\
\text { Studies }\end{array}$ & $\begin{array}{c}\text { No. of } \\
\text { Comparisons }\end{array}$ & $\begin{array}{c}\text { No. of } \\
\text { Comparisons } \\
\text { Eligible for } \\
\text { Meta-Analysis }\end{array}$ \\
\hline Audit and feedback & 103,577 & 13 & 15 & 5 \\
Case management & 2,924 & 6 & 6 & 4 \\
Clinician education & 20,806 & 18 & 20 & 10 \\
Clinician reminders & 48,614 & 40 & 48 & 36 \\
Community engagement & 23,879 & 3 & 3 & 3 \\
Continuous quality improve- & 20,097 & 9 & 9 & 3 \\
$\quad$ ment (or similar) & 35,163 & 9 & 12 & 7 \\
Delivery site change & 87,260 & 4 & 5 & 3 \\
Financial incentive (clinicians) & 16,395 & 4 & 5 & 5 \\
Financial incentive (patients) & 371,218 & 72 & 102 & 71 \\
Patient outreach & 155,726 & 26 & 28 & 23 \\
Team change & 321 & 1 & 1 & 1 \\
Visit structure change & 470,175 & 106 & 151 & 111 \\
Overall & & & & \\
\hline
\end{tabular}


the same site. Contamination is prevented by allocation at the physician, practice, or region level, which occurred in only 31 studies (40\%).

\section{Main Meta-Analyses}

There were 111 comparisons from 77 studies that contributed to the overall meta-analysis (Table 1). The pooled odds ratio expressing the effectiveness of all quality improvement interventions for either vaccination was 1.61 (95\% CI, 1.49-1.75; $\left.P<.001 ; \mathrm{I}^{2}=85 \%\right)$.

\section{Influenza Vaccination}

There were 93 comparisons from 65 studies included in meta-analyses for influenza vaccinations. The median treatment and control group vaccination rates were 0.45 ([interquartile range $[\mathrm{IQR}]=0.27$ $0.66)$, and 0.31 (IQR $=0.20-0.52)$, respectively. The odds ratio for influenza vaccination, pooled across all interventions, was 1.46 (95\% CI, 1.35-1.57; $\left.\mathrm{I}^{2}=81 \%\right)$. Fewer than 3 comparisons were available for each of community engagement, visit structure change, and continuous quality improvement-like interventions. Excluding these interventions, most components were associated with statistically significant improvements in vaccination rates (Figure 2. See also Supplemental Appendix B, Exhibit B.1 for forest plots featuring individual studies). Interventions featuring patient financial incentives $(\mathrm{OR}=1.98$, $\left.95 \% \mathrm{CI}, 1.54-2.56 ; \mathrm{I}^{2}=37 \%\right)$ and audit and feedback $\left(\mathrm{OR}=1.83,95 \% \mathrm{CI}, 1.28-2.61 ; \mathrm{I}^{2}=0 \%\right)$ were effec- tive. Patient incentives that eliminated out-of-pocket costs in a patient-pay environment ${ }^{23,24}$ appeared to be more effective than those providing a small reward in addition to preexisting third-party vaccination coverage. $^{25,26}$ Insufficient studies were available to test this hypothesis statistically, however. Audit and feedback findings were driven largely by results from the single study by Buffington et al, who were able to improve vaccination rates with regularly updated posters in physician offices tracking vaccination progress. ${ }^{27}$ Clinician reminders $(\mathrm{OR}=1.53,95 \% \mathrm{CI}, 1.26-1.85$; $\left.\mathrm{I}^{2}=71 \%\right)$, clinician financial incentives $(\mathrm{OR}=1.52,95 \%$ $\left.\mathrm{CI}, 1.20-1.93 ; \mathrm{I}^{2}=49 \%\right)$, team change $(\mathrm{OR}=1.44,95 \%$ $\left.\mathrm{CI}, 1.16-1.79 ; \mathrm{I}^{2}=67 \%\right)$, patient outreach $(\mathrm{OR}=1.42$, $\left.95 \% \mathrm{CI}, 1.30-1.55 ; \mathrm{I}^{2}=84 \%\right)$, and delivery site changes $\left(\mathrm{OR}=1.32,95 \% \mathrm{CI}, 1.14-1.52 ; \mathrm{I}^{2}=17 \%\right)$ were also associated with improvements in vaccination rates. Delivery site changes included workplace vaccination clinics ${ }^{28}$ and clinics in public housing buildings. ${ }^{29}$ These interventions were effective overall, but what elements of these were effective and where are difficult to discern, because a wide variety of intervention sites were implemented in a small number of studies. Case management and clinician education were ineffective.

\section{Pneumococcal Vaccinations}

There were 48 comparisons from 35 studies included in the meta-analyses. The median treatment and control group vaccination rates were $0.19(\mathrm{IQR}=0.11$ $0.33)$, and $0.08(\mathrm{IQR}=0.04-0.22)$, respectively. The

Figure 2. Effect of quality improvement interventions on influenza vaccination rates.

\begin{tabular}{|c|c|c|c|c|c|c|c|}
\hline \multicolumn{2}{|l|}{ Intervention } & \multirow{2}{*}{$\frac{\text { OR }(95 \% \mathrm{CI})}{3.00(1.28-7.03)}$} & \multirow{2}{*}{ Comparisons } & \multirow{2}{*}{$\begin{array}{c}\begin{array}{c}\text { Vaccinations } \\
\text { (treatment) }\end{array} \\
\text { No counts }\end{array}$} & \multirow{2}{*}{$\begin{array}{c}\begin{array}{c}\text { Vaccinations } \\
\text { (control) }\end{array} \\
\text { No counts }\end{array}$} & \multirow{2}{*}{$\begin{array}{r}\text { Patients } \\
1,426\end{array}$} & \multirow{2}{*}{$\frac{\mathbf{1}^{2}}{0}$} \\
\hline Community engagement ${ }^{\mathrm{a}}$ & $\longrightarrow \longrightarrow$ & & & & & & \\
\hline Visit structure change $^{a}$ & $\longrightarrow$ & $2.44(1.42-4.20)$ & 1 & $130 / 160$ & $103 / 161$ & 321 & 100 \\
\hline Financial incentives (patient) & $\longrightarrow$ & $1.98(1.54-2.56)$ & 5 & $652 / 1,406$ & $132 / 697$ & 8,529 & 37 \\
\hline Audit and feedback & $\longrightarrow$ & $1.83(1.28-2.61)$ & 4 & $4,490 / 6,459$ & $3,024 / 5,466$ & 12,075 & 0 \\
\hline Case management & & $1.66(0.81-3.43)$ & 4 & $381 / 668$ & $357 / 696$ & 1,364 & 67 \\
\hline Clinical reminders & 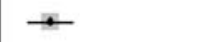 & $1.53(1.26-1.85)$ & 30 & $3,777 / 9,978$ & $2,535 / 7,720$ & 20,316 & 71 \\
\hline Financial incentives (clinical) & $\longrightarrow$ & $1.52(1.20-1.93)$ & 3 & $14,182 / 21,296$ & $10,580 / 17,608$ & 42,678 & 49 \\
\hline Team change & $\rightarrow$ & $1.44(1.16-1.79)$ & 20 & $9,001 / 32,054$ & $11,992 / 46,490$ & 81,012 & 67 \\
\hline Patient outreach & $\leftarrow$ & $1.42(1.30-1.55)$ & 59 & $30,726 / 98,570$ & $24,242 / 107,209$ & 220,981 & 84 \\
\hline Delivery site change & $\rightarrow$ & $1.32(1.14-1.52)$ & 6 & $1,857 / 2,609$ & $1,350 / 1,992$ & 10,952 & 17 \\
\hline$C Q 1^{\mathrm{a}}$ & & $0.99(0.94-1.04)$ & 2 & $396 / 654$ & $538 / 992$ & 1,646 & 0 \\
\hline \multirow[t]{2}{*}{ Clinical education } & & $0.99(0.94-1.04)$ & 8 & $1,625 / 2,359$ & $1,725 / 2,606$ & 9,326 & 0 \\
\hline & $\begin{array}{llll} & 1 & 1 \\
& & & 1 \\
& & \end{array}$ & & & & & & \\
\hline \multicolumn{8}{|c|}{$\mathrm{CQI}=$ continuous quality improvement; $\mathrm{OR}=$ odds ratio. } \\
\hline \multicolumn{8}{|c|}{$\begin{array}{l}\text { Note: Forest plot showing pooled odds ratios from random effects meta-analyses. Vaccination rates provided are crude estimates generated by summing patients } \\
\text { among studies. Many studies contributing odds ratios for meta-analysis did not provide crude counts. }\end{array}$} \\
\hline \multicolumn{8}{|c|}{ a Pooled odds ratios from fewer than 3 comparisons are reported but considered insufficient for interpretation. } \\
\hline
\end{tabular}


Figure 3. Effect of quality improvement interventions on pneumococcal vaccination rates.

\begin{tabular}{|c|c|c|c|c|c|c|c|}
\hline \multicolumn{2}{|l|}{ Intervention } & OR $(95 \% \mathrm{Cl})$ & Comparisons & $\begin{array}{l}\text { Vaccinations } \\
\text { (treatment) }\end{array}$ & $\begin{array}{l}\text { Vaccinations } \\
\text { (control) }\end{array}$ & Patients & $\mathbf{I}^{2}$ \\
\hline Financial incentives (clinical) ${ }^{c}$ & $\longrightarrow$ & $7.43(2.25-24.53)$ & 1 & No counts & No counts & 1,914 & 100 \\
\hline Visit structure change $^{a}$ & $\rightarrow$ & $2.25(1.30-3.92)$ & 1 & $53 / 160$ & 29/161 & 321 & 100 \\
\hline Clinical reminders & $\longrightarrow$ & $2.13(1.50-3.03)$ & 27 & $2,123 / 6,886$ & $919 / 6,742$ & 36,631 & 75 \\
\hline Team change & $\longrightarrow$ & $2.09(1.48-2.95)$ & 14 & $801 / 2,086$ & $626 / 1,740$ & 10,228 & 51 \\
\hline$\left.\mathrm{CQ}\right|^{\mathrm{a}}$ & 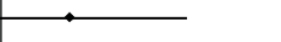 & $1.86(0.66-5.21)$ & 2 & No Counts & No Counts & 4,725 & 83 \\
\hline Patient outreach & $\rightarrow$ & $1.80(1.54-2.11)$ & 26 & $572 / 1,999$ & $406 / 1,700$ & 66,301 & 67 \\
\hline Community engagement ${ }^{\mathrm{a}}$ & 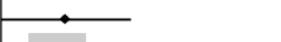 & $1.78(1.00-3.17)$ & 2 & No Counts & No Counts & 23,699 & 78 \\
\hline Delivery site change & - & $1.66(1.59-1.74)$ & 1 & No Counts & No Counts & No Counts & 100 \\
\hline Clinical education & $\longrightarrow$ & $1.54(1.19-1.99)$ & 7 & $478 / 1,715$ & $301 / 1,362$ & 7,665 & 72 \\
\hline Case management & $\longrightarrow$ & $1.49(1.05-2.13)$ & 3 & $167 / 668$ & $112 / 696$ & 1,364 & 0 \\
\hline \multirow[t]{2}{*}{ Audit and feedback } & 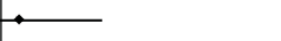 & $1.18(0.57-2.45)$ & 3 & $477 / 653$ & $475 / 650$ & 3,440 & 7 \\
\hline & \begin{tabular}{l|l|l} 
& 1 \\
& & 10
\end{tabular} & & & & & & \\
\hline
\end{tabular}

$\mathrm{CQI}=$ continuous quality improvement; $\mathrm{OR}=$ odds ratio.

Note: Forest plot showing pooled odds ratios from random effects meta-analyses. Vaccination rates provided are crude estimates generated by summing patients among studies. Many studies contributing odds ratios for meta-analysis did not provide crude counts.

a Pooled odds ratios from fewer than 3 comparisons are reported, but considered insufficient for interpretation. No comparisons involving patient financial incentives were available for meta-analysis.

odds ratio for pneumococcal vaccinations, pooled across all interventions, was 2.01 (95\% CI, 1.72-2.36; $\left.\mathrm{I}^{2}=72 \%\right)$. Three or more comparisons were available for clinician reminders, team change, patient outreach, clinician education, case management, and audit and feedback. Except for audit and feedback $(\mathrm{OR}=1.18$, $\left.95 \% \mathrm{CI}, 0.57-2.45 ; \mathrm{I}^{2}=7 \%\right)$, these interventions were associated with improvements in vaccination rates (Figure 3. See also Supplemental Appendix B, Exhibit B.2 for forest plots featuring individual studies). Interventions featuring clinician reminders $(\mathrm{OR}=2.13,95 \% \mathrm{CI}$, $\left.1.50-3.03 ; \mathrm{I}^{2}=75 \%\right)$, team change $(\mathrm{OR}=2.09,95 \% \mathrm{CI}$, $\left.1.48-2.95 ; \mathrm{I}^{2}=51 \%\right)$, and patient outreach $(\mathrm{OR}=1.80$, $\left.95 \% \mathrm{CI}, 1.54-2.11 ; \mathrm{I}^{2}=67 \%\right)$ had the highest odds ratios. Clinician education $(\mathrm{OR}=1.54,95 \% \mathrm{CI}, 1.19$ $\left.1.99 ; \mathrm{I}^{2}=72 \%\right)$ and case management $(\mathrm{OR}=1.49$, $\left.95 \% \mathrm{CI}, 1.05-2.13 ; \mathrm{I}^{2}=0 \%\right)$ were also associated with improvements in pneumococcal vaccination rates.

\section{Meta-Analyses Within Intervention Substrata}

Interventions featuring clinician reminders, team change, and patient outreach had moderate to high heterogeneity and sufficient comparisons for substratification. For clinician reminders, most heterogeneity was explained by declining odds ratios with time. For patient outreach and team change, the results of metaanalyses within intervention substrata are presented in Table 2 (forest plots are available in Supplemental qualification. Among patient outreach strategies for influenza, community media campaigns appeared most effective. This finding should be interpreted cautiously, however, because studies took place in settings with relatively captive audiences and a high prevalence of high-risk patients (eg, seniors' centers). ${ }^{29,30}$ For pneumococcal vaccination, the pooled odds ratio for waiting and examination room posters may also be misleadingly high, because there were few comparisons $(n=5)$, and the highest performing comparisons combined posters with other effective interventions..$^{31,32}$ In 2 studies that considered them alone, waiting and examination room posters were not significantly associated with vaccination rates. ${ }^{31,32}$

Generally, outreach methods involving personal contact with patients achieved higher pooled odds ratios. For influenza vaccinations, the most effective intervention, excepting community media campaigns, was telephone reminders delivered by clinic staff. For pneumococcal vaccinations, office brochures handed out to eligible patients by clinic staff before their appointments was most effective. Meta-regression detected significant differences between pneumococcal vaccination outreach strategies. Office brochures at the point of care were 3.87 times more effective than mailed reminders, whereas community media campaigns, patient-held preventive care checklists, and waiting or examination room posters were, respectively, $0.85,0.77$, and 0.75 times less effective than mailed reminders. Among team change interventions 
Table 2. Results of Meta-Analyses Within Substrata of Patient Outreach and Team Change

\begin{tabular}{|c|c|c|c|c|c|}
\hline Influenza Vaccination & $\begin{array}{c}\text { Pooled OR } \\
(95 \% \mathrm{Cl})\end{array}$ & $1^{2}$ & Pneumococcal Vaccination & $\begin{array}{c}\text { Pooled OR } \\
(95 \% \mathrm{Cl})\end{array}$ & $\mathbf{I}^{2}$ \\
\hline Patient outreach medium & & & Patient outreach medium & & \\
\hline Community media campaign ${ }^{a}$ & $\begin{array}{c}3.16 \\
(1.35-7.37)\end{array}$ & 0 & Emergency medical technician outreach ${ }^{b}$ & $\begin{array}{c}8.65 \\
(0.02-4899.87)\end{array}$ & 100 \\
\hline Telephone reminders ${ }^{a}$ & $\begin{array}{c}2.74 \\
(1.23-6.12)\end{array}$ & 67 & Brochures at office visit ${ }^{a}$ & $\begin{array}{c}5.86 \\
(3.29-10.44)\end{array}$ & 0 \\
\hline Waiting/examination room posters ${ }^{b}$ & $\begin{array}{c}1.78 \\
(0.53-6.01)\end{array}$ & 95 & Telephone reminders ${ }^{b}$ & $\begin{array}{c}2.86 \\
(2.31-3.56)\end{array}$ & 0 \\
\hline Mailed print materiala & $\begin{array}{c}1.45 \\
(1.30-1.61)\end{array}$ & 89 & Waiting/examination room posters ${ }^{\mathrm{a}}$ & $\begin{array}{c}1.92 \\
(1.09-3.40)\end{array}$ & 57 \\
\hline Brochures at office visit ${ }^{b}$ & $\begin{array}{c}1.38 \\
(0.82-2.33)\end{array}$ & 0 & Mailed print material ${ }^{a}$ & $\begin{array}{c}1.66 \\
(1.59-1.74)\end{array}$ & 0 \\
\hline Patient-held preventive care schedule & $\begin{array}{c}1.28 \\
(0.82-1.99)\end{array}$ & 53 & Home visit education ${ }^{b}$ & $\begin{array}{c}1.52 \\
(0.74-3.11)\end{array}$ & 100 \\
\hline Home visit education ${ }^{b}$ & $\begin{array}{c}0.94 \\
(0.64-1.40)\end{array}$ & 0 & Community media campaign ${ }^{a}$ & $\begin{array}{c}1.31 \\
(1.28-1.55)\end{array}$ & 0 \\
\hline Emergency medical technician outreach ${ }^{b}$ & $\begin{array}{c}0.67 \\
(0.01-36.06)\end{array}$ & 100 & Patient-held preventive care schedule ${ }^{a}$ & $\begin{array}{c}1.29 \\
(1.06-1.57)\end{array}$ & 0 \\
\hline Team change: type of additional personnel & & & Team change: type of additional personnel & & \\
\hline Multidisciplinary teamb & $\begin{array}{c}2.44 \\
(1.42-4.20)\end{array}$ & 100 & Emergency medical technician & $\begin{array}{c}8.65 \\
(0.02-4899.87)\end{array}$ & 100 \\
\hline Nurse, autonomous vaccinations ${ }^{a}$ & $\begin{array}{c}1.63 \\
(1.30-2.04)\end{array}$ & 7 & Nurse, autonomous vaccinations ${ }^{b}$ & $\begin{array}{c}7.03 \\
(2.98-16.57)\end{array}$ & 0 \\
\hline Nurse, no autonomous vaccinations & $\begin{array}{c}1.14 \\
(0.88-1.48)\end{array}$ & 60 & Multidisciplinary team ${ }^{b}$ & $\begin{array}{c}2.25 \\
(1.30-3.92)\end{array}$ & 100 \\
\hline Pharmacist & $\begin{array}{c}1.11 \\
(0.62-1.98)\end{array}$ & 0 & Nurse, no autonomous vaccinations ${ }^{a}$ & $\begin{array}{c}1.96 \\
(1.28-3.03)\end{array}$ & 60 \\
\hline Emergency medical technician ${ }^{b}$ & $\begin{array}{c}0.67 \\
(0.01-36.06)\end{array}$ & 100 & Pharmacist & $\begin{array}{c}1.03 \\
(0.62-1.74)\end{array}$ & 0 \\
\hline \multicolumn{6}{|l|}{$\mathrm{OR}=$ odds ratio. } \\
\hline \multicolumn{6}{|c|}{ Note: See forest plots in Supplemental Appendix C for more detail. } \\
\hline \multicolumn{6}{|l|}{ a Pooled odds ratios significant at $P<.05$. } \\
\hline
\end{tabular}

for influenza vaccinations, we found that having nurses assume responsibility for administering vaccinations was effective, whereas interventions in which nurses or pharmacists assessed patients and reminded physicians, but did not themselves administer vaccinations, were ineffective. We were unable to examine this relationship in studies of pneumococcal vaccinations because of insufficient comparisons.

\section{Numbers Needed to Treat}

Results for effective quality improvement strategies are summarized as numbers needed to treat (NNT), assuming baseline levels of vaccination similar to those reported in community studies of elderly adults ${ }^{8}$ (Table 3 and Supplemental Appendix C, Exhibit C.5).

\section{Sensitivity Analyses and Publication Bias}

Randomized study design was not significantly associated with study odds ratios within intervention strata. After excluding 2 clear outlier studies, ${ }^{33,34}$ quality score was also not significantly associated with study odds ratios for any intervention. Funnel plots showed higher odds ratios in smaller studies. Harbord's test was positive for small study effects among studies of patient outreach for influenza and pneumococcal vaccinations and of team change for influenza vaccinations. These findings suggest potential publication bias.

\section{DISCUSSION}

We reviewed the evidence for effectiveness of quality improvement interventions for increasing influenza and pneumococcal vaccination rates. Most interventions were associated with modest improvements in vaccination rates.

Team change, patient outreach, and clinician reminders were effective for both influenza and pneumococcal vaccinations. We found that interventions involving team change were effective, especially where nurses had been assigned responsibilities for administering vaccine. Configuring additional personnel so that they are able relieve physicians of vaccinations 
seems important to successful team change. ${ }^{14}$ Additionally, patient outreach may better increase vaccinations to the extent that direct personal contact is achieved. A previous review has similarly reported that reminders involving person-to-person telephone contact were most effective. ${ }^{10}$

Clinician reminders and education were associated with greater improvements for pneumococcal than for influenza vaccinations. Awareness and support may be less common for pneumococcal ${ }^{35}$ than for influenza vaccinations, ${ }^{36-38}$ making pneumococcal vaccinations relatively low hanging fruit. Audit and feedback appeared effective for influenza, but not pneumococcal, vaccinations. Audit and feedback may have been effective for influenza vaccinations because of the prominent tracking posters used in Buffington et al. ${ }^{27}$ The use of materials with high visual appeal and clarity has been previously associated with increased

\section{Table 3. Numbers Needed to Treat to Obtain an Additional Vaccination}

\begin{tabular}{|c|c|}
\hline Characteristics & NNT \\
\hline \multicolumn{2}{|l|}{ Influenza vaccinations } \\
\hline \multicolumn{2}{|l|}{ Baseline vaccination rate of $70 \%$ assumed } \\
\hline Patient outreach (community media) & 6 \\
\hline Patient outreach (telephone reminders) & 6 \\
\hline Financial incentives, patient & 8 \\
\hline Audit and feedback & 9 \\
\hline Team change (nurse vaccine administration) & 11 \\
\hline Clinician reminders & 12 \\
\hline Financial incentives, clinician & 13 \\
\hline Patient outreach (mailed print materials) & 14 \\
\hline Team change (overall) & 14 \\
\hline Patient outreach (overall) & 15 \\
\hline Delivery site change & 18 \\
\hline \multicolumn{2}{|l|}{ Pneumococcal vaccinations } \\
\hline \multicolumn{2}{|l|}{ Baseline vaccination rate of $60 \%$ assumed } \\
\hline $\begin{array}{l}\text { Patient outreach (brochures handed out } \\
\text { before appointments) }\end{array}$ & 3 \\
\hline Clinician reminders & 6 \\
\hline Team change (overall) & 6 \\
\hline $\begin{array}{l}\text { Team change (nurses without vaccine adminis- } \\
\text { tration responsibilities) }\end{array}$ & 7 \\
\hline $\begin{array}{l}\text { Patient outreach (waiting/examination room } \\
\text { posters) }\end{array}$ & 7 \\
\hline Patient outreach (overall) & 8 \\
\hline Clinician education & 9 \\
\hline Patient outreach (mailed print materials) & 9 \\
\hline Case management & 11 \\
\hline Patient outreach (community media) & 13 \\
\hline Patient outreach (preventive care checklists) & 17 \\
\hline \multicolumn{2}{|l|}{ NNT = number needed to treat. } \\
\hline \multicolumn{2}{|c|}{$\begin{array}{l}\text { Note: Interventions included in this table had summary odds ratios statistically } \\
\text { greater than } 1.0(P<.05) \text { based on } 3 \text { or more studies. Numbers needed to } \\
\text { treat are provided assuming other baseline vaccination rates in Supplemental } \\
\text { Appendix C, Exhibit C.5. }\end{array}$} \\
\hline
\end{tabular}

vaccination rates. ${ }^{14}$ Clinician and patient financial incentives were both effective for influenza vaccinations, but they could not be evaluated for pneumococcal vaccinations. The 2 successful studies of patient financial incentives took place in out-of-pocket payment environments. ${ }^{23,24}$ Where demand for vaccinations is not pent up by inability to pay, the benefit of patient incentives may be smaller. ${ }^{25,26}$ Case management, surprisingly, was not very effective-possibly because case managers may have prioritized other disease-related process of care.

Several limitations of our review should be borne in mind. Our funnel plots and associated tests suggested publication bias, which may have led our pooled odds ratios to be overly optimistic. Our review also did not address the economic value of the interventions. Additionally, the included studies may not generalize well to nonelderly adults or adults not in a physician's care, for whom vaccination recommendations have recently been expanded. ${ }^{7}$

More importantly, we have taken a highly inclusive approach toward meta-analysis. There are 2 major limitations of this approach. First, our analysis of Downs and Black items identified a high prevalence of design or reporting flaws in the included studies. Lack of blinding may be relatively unimportant for quality improvement interventions designed to act, in part, by increasing awareness of vaccinations and for outcomes that can be measured relatively objectively by reviewing charts or billing data. Only $60 \%$ of studies reported and accounted adequately for potential confounders, however. This proportion was higher in randomized than in observational studies.

We have nonetheless reported odds ratios pooled from all studies. Neither randomization nor Downs and Black scores were associated with significant differences in odds ratios. The inclusion of a wide range of studies allowed us to produce quantitative summaries for many intervention categories. In particular, interventions requiring policy support or action on a community scale, such as audit and feedback and community media campaigns, are difficult to randomizeobservational studies comprise an important source of insight. ${ }^{39}$ Our study quality tables (Supplemental Appendix B) provide further detail on methodological issues for potential users.

Second, many of our pooled estimates contained residual heterogeneity. Our ability to explore heterogeneity was limited by lack of evidence. ${ }^{40}$ For example, reasons for decreases in the effectiveness of clinician reminders in recent years are unknown. We have incorporated heterogeneity into our meta-analysis by using a random-effects approach. Users should interpret pooled odds ratios as estimates of the average 
intervention effect, as opposed to a single, true effect. Our 95\% confidence limits may provide bounds on the expected performance of the intervention under most circumstances. In any event, a single true effect would not likely be useful, because most users can identify mitigating or potentiating factors unique to their circumstances. Our estimates provide a preliminary basis for selecting interventions; potential users should examine our summaries of individual studies (Supplemental Appendix A. Exhibit A.5) and intervention-specific forest plots (Supplemental Appendix B) in light of their own circumstances and a theoretical understanding of behavior change. ${ }^{41,42}$

Building on previous reviews, we have produced a comprehensive, quantitative summary of the effectiveness of interventions to improve influenza and pneumococcal vaccination rates. Our results suggest that (1) shifting vaccine administration from physicians to members of the primary care team with clear responsibilities for chronic and preventive care and (2) activating patients through personal outreach may stand the best chance of improving vaccination rates in community dwelling adults. Nonetheless, practitioners and policy makers should temper their expectations of quality improvement interventions. In few treatment arms had vaccination rates improved sufficiently to meet national policy targets. ${ }^{43,44}$ Further research is required to develop and evaluate more potent approaches and to better understand how and why they work.

To read or post commentaries in response to this article, see it online at http://www.annfammed.org/content/10/6/538.

Key words: vaccination; influenza; Streptococcus pneumoniae; quality improvement; primary health care

Submitted October 24, 2011, submitted, revised, February 8, 2012; accepted March 2, 2012.

Funding support: This work was funded in part by studentships, salary awards, and operating grants from Alberta Innovates - Health Solutions, Canadian Institute of Health Research (Institute of Nutrition, Metabolism, and Diabetes), and the Canada Research Chairs. Mr Lau was funded by MD/PhD studentships from the Canadian Institutes of Health Research (CIHR) and Alberta Innovates - Health Solutions (AIHS). Dr Majumdar is a health scholar with Al-HS. Dr Johnson is a senior scholar with Al-HS and holds a Canada Research Chair in Diabetes Health Outcomes. This work was supported in part by a grant from Alberta Health and Wellness and a CIHR Team Grant to the Alliance for Canadian Health Outcomes Research in Diabetes (ACHORD) (reference \#: OTG-88588), sponsored by the CIHR Institute of Nutrition, Metabolism and Diabetes.

Previous presentations: This study was presented orally at the Annual Conference of the Canadian Association for Population Therapeutics, Ottawa, Ontario, April 17-19, 2011, and as a poster at the International Conference of the International Society for Pharmacoepidemiology, Chicago, Illinois, August 14-172011.

\section{References}

1. Thompson MG, Shay DK, Zhou H, et al; Centers for Disease Control and Prevention (CDC). Estimates of deaths associated with seasonal influenza-United States, 1976-2007. MMWR Morb Mortal Wkly Rep. 2010;59(33):1057-1062.

2. Thompson WW, Shay DK, Weintraub E, et al. Influenza-associated hospitalizations in the United States. JAMA. 2004;292(11):1333-1340.

3. Pulido M, Sorvillo F. Declining invasive pneumococcal disease mortality in the United States, 1990-2005. Vaccine. 2010;28(4):889-892.

4. Nuorti JP, Whitney CG; Centers for Disease Control and Prevention (CDC); Advisory Committee on Immunization Practices. Updated recommendations for prevention of invasive pneumococcal disease among adults using the 23-valent pneumococcal polysaccharide vaccine (PPSV23). MMWR Morb Mortal Wkly Rep. 2010;59(34):1102-1106.

5. Advisory Committee on Immunization Practices. Prevention of Pneumococcal Disease: Recommendations of the Advisory Committee on Immunization Practices (ACIP). MMWR Morb Mortal Wkly Rep. 1997;46(RR-8):1-31.

6. Advisory Committee on Immunization Practices. Prevention and control of seasonal influenza with vaccines: Recommendations of the Advisory Committee on Immunization Practices (ACIP), 2009. MMWR Morb Mortal Wkly Rep. 2009;58(RR-8):1-52.

7. Advisory Committee on Immunization Practices. Prevention and control of influenza with vaccines. MMWR Morb Mortal W/kly Rep. 2010;59(rr08):1-62.

8. Euler GL, Lu PJ, Shefer A, et al. Influenza vaccination coverage among children and adults-United States, 2008-09 influenza season. JAMA. 2009;302(19):2085-2086.

9. Bardenheier BH, Wortley PM, Euler G; Centers for Disease Control and Prevention (CDC). Influenza and pneumococcal vaccination coverage among persons aged $\geq 65$ years and persons aged 18-64 years with diabetes or asthma-United States, 2003. MMWR Morb Mortal Wkly Rep. 2004;53(43):1007-1012.

10. Jacobson VJ, Szilagyi P. Patient reminder and patient recall systems to improve immunization rates. Cochrane Database Syst Rev. 2005; 20;(3):CD003941.

11. Guide to Community Preventive Services. Vaccinations to prevent diseases: universally recommended vaccinations. Community Guide to Preventive Services. 2010:1-2.

12. Guide to Community Preventive Services. Vaccinations to prevent diseases: targeted vaccinations. Community Guide to Preventive Services. 2010:1-2. http://www.thecommunityguide.org/vaccines/ targeted/index.html.

13. Ndiaye SM, Hopkins DP, Shefer AM, et al. Task Force on Community Preventive Services. Interventions to improve influenza, pneumococcal polysaccharide, and hepatitis B vaccination coverage among high-risk adults: a systematic review. Am J Prev Med. 2005;28(5)(Suppl):248-279.

14. Stone EG, Morton SC, Hulscher ME, et al. Interventions that increase use of adult immunization and cancer screening services: a meta-analysis. Ann Intern Med. 2002;136(9):641-651.

15. Thomas RE, Russell ML, Lorenzetti DL. Systematic review of interventions to increase influenza vaccination rates of those 60 years and older. Vaccine. 2010;28(7):1684-1701.

16. Downs SH, Black N. The feasibility of creating a checklist for the assessment of the methodological quality both of randomised and non-randomised studies of health care interventions. J Epidemiol Community Health. 1998;52(6):377-384.

17. Shojania KG, McDonald KM, Wachter RM, Owens DK. Closing the quality gap. Technical Review, No. 9: Vol. I: Series Overview and Methodology. AHRQ. 2004:1-85.

18. Shojania KG, Ranji SR, McDonald KM, et al. Effects of quality improvement strategies for type 2 diabetes on glycemic control: a meta-regression analysis. JAMA. 2006;296(4):427-440. 
19. Campbell MK, Mollison J, Grimshaw JM. Cluster trials in implementation research: estimation of intracluster correlation coefficients and sample size. Stat Med. 2001;20(3):391-399.

20. Gulliford MC, Ukoumunne OC, Chinn S. Components of variance and intraclass correlations for the design of community-based surveys and intervention studies: data from the Health Survey for England 1994. Am J Epidemiol. 1999;149(9):876-883.

21. Harbord R, Harris R, Sterne J. Updated tests for smal-study effects in meta-analysis. Stata J. 2009;9(2):197-210.

22. Sterne J. Meta-analysis in Stata: An Updated Collection From the Stata Journal. College Station, TX: Stata Press; 2009.

23. Nexøe J, Kragstrup J, Rønne T. Impact of postal invitations and user fee on influenza vaccination rates among the elderly. A randomized controlled trial in general practice. Scand J Prim Health Care. 1997;15(2):109-112.

24. Satterthwaite P. A randomised intervention study to examine the effect on immunisation coverage of making influenza vaccine available at no cost. N Z Med J. 1997;110(1038):58-60.

25. Moran WP, Nelson K, Wofford JL, Velez R, Case LD. Increasing influenza immunization among high-risk patients: education or financial incentive? Am J Med. 1996;101(6):612-620.

26. Nowalk MP, Lin CJ, Toback SL, et al. Improving influenza vaccination rates in the workplace: a randomized trial. Am J Prev Med. 2010;38(3):237-246

27. Buffington J, Bell KM, LaForce FM; Genesee Hospital Medical Staff. A target-based model for increasing influenza immunizations in private practice. J Gen Intern Med. 1991;6(3):204-209.

28. Ahmed F, Friedman C, Franks A, et al. Effect of the frequency of delivery of reminders and an influenza tool kit on increasing influenza vaccination rates among adults with high-risk conditions. Am J Manag Care. 2004;10(10):698-702.

29. Schensul JJ, Radda K, Coman E, Vazquez E. Multi-level intervention to prevent influenza infections in older low income and minority adults. Am J Community Psychol. 2009;43(3-4):313-329.

30. Leirer VO, Morrow DG, Pariante G, Doksum T. Increasing influenza vaccination adherence through voice mail. J Am Geriatr Soc. 1989;37(12):1147-1150.

31. Thomas DM, Ray SM, Morton FJ, et al. Patient education strategies to improve pneumococcal vaccination rates: randomized trial. J Investig Med. 2003;51(3):141-148.

32. Latessa RA, Cummings DM, Lilley SH, Morrissey SL. Changing practices in the use of pneumococcal vaccine. Fam Med. 2000;32(3):196-200.
33. Harris RP, O'Malley MS, Fletcher SW, Knight BP. Prompting physicians for preventive procedures: a five-year study of manual and computer reminders. Am J Prev Med. 1990;6(3):145-152.

34. Lennox N, Bain C, ReyConde T, et al. Cluster randomized-controlled trial of interventions to improve health for adults with intellectual disability who live in private dwellings. J Appl Res Intellect Disabil. 2010;23(4):303-311.

35. Mieczkowski TA, Wilson SA. Adult pneumococcal vaccination: a review of physician and patient barriers. Vaccine. 2002;20(9-10): 1383-1392.

36. Davis MM, McMahon SR, Santoli JM, Schwartz B, Clark SJ. A national survey of physician practices regarding influenza vaccine. J Gen Intern Med. 2002;17(9):670-676.

37. Nichol KL, Zimmerman R. Generalist and subspecialist physicians' knowledge, attitudes, and practices regarding influenza and pneumococcal vaccinations for elderly and other high-risk patients: a nationwide survey. Arch Intern Med. 2001;161(22):2702-2708.

38. Szilagyi PG, Shone LP, Barth R, et al. Physician practices and attitudes regarding adult immunizations. Prev Med. 2005;40(2):152-161.

39. Norris SL, Atkins D. Challenges in using nonrandomized studies in systematic reviews of treatment interventions. Ann Intern Med. 2005;142(12 Pt 2):1112-1119.

40. Shepperd S, Lewin S, Straus S, et al. Can we systematically review studies that evaluate complex interventions? PLoS Med. 2009;6(8): e1000086.

41. Looijmans-van den Akker I, Hulscher ME, Verheij TJ, RiphagenDalhuisen J, van Delden JJ, Hak E. How to develop a program to increase influenza vaccine uptake among workers in health care settings? Implement Sci. 2011;6:47.

42. Eccles M, Grimshaw J, Walker A, Johnston M, Pitts N. Changing the behavior of healthcare professionals: the use of theory in promoting the uptake of research findings. J Clin Epidemiol. 2005;58(2):107-112.

43. Centers for Disease Control and Prevention. Immunization and infections diseases. In: Healthy People 2010. Atlanta, GA: CDC; 2000:14-1, 14-59.

44. Public Health Agency of Canada. Final report of outcomes from the National Consensus Conference for Vaccine-Preventable Diseases in Canada, June 12-14, 2005, Quebec City, Quebec. Can Commun Dis Rep. 2008;34(Suppl 2):1-56 